\title{
Concurrent chemoradiation for resected gall bladder cancers and cholangiocarcinomas
}

\author{
Muhammad M. Fareed ${ }^{1}$, Lyudmila DeMora ${ }^{2}$, Nestor F. Esnaola ${ }^{3}$, Crystal S. Denlinger ${ }^{4}$, Andreas Karachristos ${ }^{3}$, \\ Eric E. Ross ${ }^{2}$, John Hoffman ${ }^{3}$, Joshua E. Meyer ${ }^{5}$ \\ ${ }^{1}$ Department of Radiation Oncology, Dana-Farber/Brigham and Women's Cancer Center, Harvard Medical School, Boston, MA, USA; ${ }^{2}$ Department \\ of Biostatistics, ${ }^{3}$ Department of Surgical Oncology, ${ }^{4}$ Department of Hematology/Oncology, ${ }^{5}$ Department of Radiation Oncology, Fox Chase Cancer \\ Center-Temple Health, Philadelphia, PA, USA \\ Contributions: (I) Conception and design: MM Fareed; (II) Administrative support: JE Meyer; (III) Provision of study materials or patients: J Hoffman, \\ NF Esnaola, A Karachristos; (IV) Collection and assembly of data: JE Meyer, L DeMora, EE Ross; (V) Data analysis and interpretation: L DeMora, \\ EE Ross; (VI) Manuscript writing: All authors; (VII) Final approval of manuscript: All authors. \\ Correspondence to: Muhammad M. Fareed. Department of Radiation Oncology, Dana-Farber/Brigham and Women's Cancer Center, Harvard \\ Medical School, Boston, MA, USA. Email: mmfareed@bwh.harvard.edu.
}

Background: Gallbladder cancer (GBC) and cholangiocarcinoma (CCA) are rare entities with relatively poor prognoses. We compared treatment outcomes of definitive resection with or without neoadjuvant therapy in GBC and CCA patients.

Methods: All non-metastatic GBC and CCA patients at a single institution who underwent definitive resection from 1992-2016 were analyzed. We compared overall survival (OS), locoregional failure (LRF) and distant failure (DF) in patients who received neoadjuvant therapy (chemotherapy and/or radiation) versus those who did not receive neoadjuvant treatment. OS was analyzed using the Kaplan-Meier method and log rank tests. Cox proportional hazard models were used to analyze time to recurrence.

Results: Out of 128 patients, 90 had GBC and 38 had CCA, 25 patients (27\%) among GBC and 8 patients (21\%) with CCA were T3, T4 or node positive. Overall, 52 (40\%) GBC and 25 (20\%) CCA patients received neoadjuvant treatment, chemotherapy alone 60 patients $(47 \%)$ or radiation with or without chemotherapy 17 patients (13\%). Chemotherapy was single agent in 44 patients (34\%) and multiagent in 25 (20\%). The median OS for GBC patients was 3.1 years with 2.6 years for no neoadjuvant group and 3.1 years for neoadjuvant group $(\mathrm{P}=0.6786)$. Median OS was 2.6 years for CCA patients, 3.6 years for no neoadjuvant therapy versus 2.0 years for neoadjuvant group $(\mathrm{P}=0.1613)$. There was a trend towards increased DF in patients with CCA and GBC receiving neoadjuvant therapy: HR 2.74, 95\% CI, 0.73-10.3, $\mathrm{P}=0.14$ and $0.92,95 \% \mathrm{CI}, 0.44-1.93, \mathrm{P}=0.82$ respectively. The hazard ratio for time to LRF in CCA patients receiving neoadjuvant treatment was $3.17,95 \% \mathrm{CI}, 0.62-16.31, \mathrm{P}=0.16$ whereas HR was $0.15,95 \%$ CI, $0.10-1.76, \mathrm{P}=0.23$ for GBC patients. Among GBC patients, the pattern of first failure was locoregional in $8(10 \%)$ having $3 \mathrm{LRF}$ in neoadjuvant group ( 2 with chemotherapy, 1 with CRT, 0 with RT alone) as compared to 5 in adjuvant group. Among 28 (35\%) patients with DF first, 15 patients received neoadjuvant therapy versus 13 patients in non-neoadjuvant group. In CCA patients, LRF occurred first in 6 patients receiving neoadjuvant treatment ( 3 with chemotherapy, 1 with CRT, 2 with RT alone) as compared to 2 patients who were treated with non-neoadjuvant CRT. DF was the first site of failure in 9 patients treated with neoadjuvant CRT (8 with chemotherapy, 0 with CRT and 1 with RT alone) as compared to 4 patients without neoadjuvant treatment.

Conclusions: In this retrospective data set, a trend towards better survival was seen in adjuvantly treated CCA patients, but not in GBC patients. Recurrence patterns also appear different among the two, which might be attributed to treatment modality used, patient selection or unmeasured factors.

Keywords: Gallbladder cancer (GBC); cholangiocarcinoma (CCA); neoadjuvant; resection; chemoradiation; chemotherapy

(C) Journal of Gastrointestinal Oncology. All rights reserved. 
Submitted Mar 16, 2018. Accepted for publication May 02, 2018.

doi: 10.21037/jgo.2018.05.09

View this article at: http://dx.doi.org/10.21037/jgo.2018.05.09

\section{Introduction}

Biliary tract (gallbladder and bile ducts) cancers are uncommon, accounting for about 11,740 cases diagnosed annually in the United States (1). Being rare biliary tract cancers (BTC), cholangiocarcinomas (CCAs) are often challenging in terms of diagnosing and treating (2). These are highly fatal cancers because of being locally advanced at presentation and their high proclivity for distant metastases with $5-15 \% 5$-year overall survival (OS) rates $(3,4)$. Surgery is only feasible upfront in about $35 \%$ of patients because of unresectable disease at presentation (5). High rates of both local and distant failure (DF) have prompted interest in neoadjuvant chemotherapy and radiation therapy (RT).

There is a growing interest in a notion that neoadjuvant chemoradiation may convert unresectable disease into resectable and may prolong survival. In a series of 157 biliary tract malignancies from MD Anderson Cancer Center, neither neoadjuvant nor adjuvant treatment improved survival in patients undergoing surgical resection with $1 \mathrm{~cm}$ tumor free margins (6). However, neoadjuvant chemoradiation has shown promising local control rates without demonstrating improved long term survival (7). In a report of 45 patients undergoing concurrent chemoradiotherapy (CRT) in resected extrahepatic CCA, the benefit for neoadjuvant CRT was shown by longer 5 -year survival ( $53 \%$ vs. $23 \%$ ) and similar grade 2 to 3 surgical morbidity (16\% vs. $33 \%$ ) compared with those treated postoperatively. In this series, among 12 patients who were treated neoadjuvantly, 3 had a complete pathologic response and 11 were able to undergo a complete (R0) resection despite having more advanced disease at presentation (8).

In this study, we reviewed treatment outcomes of definitive resection with or without neoadjuvant therapy in BTCs. We hypothesized that current neoadjuvant treatment would improve the survival probability after resection of BTC with adequate negative margins.

\section{Methods}

After approval from our Institutional Review Board, we retrospectively analyzed all patients of 18 years or elder with gallbladder cancer (GBC) and CCA who underwent definitive resection from January 01, 1992 to June 30, 2016. CCA was classified as intrahepatic or extrahepatic depending on anatomic location. Only patients with histologically confirmed gallbladder adenocarcinoma and CCA were included. Patients with metastatic disease at presentation and those who did not undergo operative exploration for whatsoever reason were excluded. In patients who were medically fit to undergo resection underwent surgery with curative intent. Resection was considered potentially curative if all gross disease was resected successfully. Palliative chemoradiation was considered in patients with unresectable disease. Data abstracted included demographic information, stage, treatment (chemotherapy and/or RT) and outcome after surgery. We compared OS, locoregional failure (LRF) and DF in patients who received neoadjuvant therapy (chemotherapy and/or radiation) versus those who did not receive neoadjuvant treatment. All neoadjuvant treatment patients (chemotherapy alone, CRT or RT alone) were combined for final analysis due to small numbers in each group. Patient and treatment characteristics are summarized in Table 1.

Data was analyzed using frequencies, percentages, means, medians, and standard deviations. Group characteristics were compared using Chi-square tests, Fisher's exact tests, Wilcoxon rank-sum tests, Kruskal-Wallis tests, or Spearman's correlations as appropriate. We analyzed trends in OS by creating Kaplan-Meier survival curves and testing for differences between groups using logrank tests. We used Cox proportional hazard models to control for potential confounders. In our main analysis, we compared neoadjuvant and adjuvant treatment groups. We assumed that $50 \%$ of patients will receive adjuvant treatment. Assuming a median survival of 18 months in the adjuvant treatment group, we had at least $80 \%$ power to detect a difference between this group and the neoadjuvant treatment group if the median survival for these patients is 36 months. These calculations use the method of Lakatos and assume uniform accrual over 21.5 years, with $5 \%$ type-I error for a 2-sided test (9). In secondary analysis, we used multivariate logistic regression models to examine failure following surgical resection. 
Table 1 Patient and treatment characteristics

\begin{tabular}{|c|c|}
\hline Characteristics & Patients, n (\%) \\
\hline \multicolumn{2}{|l|}{ Age, years } \\
\hline Median & 68.5 \\
\hline Range & $35-93$ \\
\hline \multicolumn{2}{|l|}{ Gender } \\
\hline Male & $45(35.0)$ \\
\hline Female & $83(65.0)$ \\
\hline \multicolumn{2}{|l|}{ Race } \\
\hline African American & $10(7.8)$ \\
\hline Caucasian & $103(80.5)$ \\
\hline Other & $15(11.7)$ \\
\hline \multicolumn{2}{|l|}{ Primary disease site } \\
\hline Gallbladder & $90(70.0)$ \\
\hline Bile duct & $38(30.0)$ \\
\hline \multicolumn{2}{|l|}{ Performance status } \\
\hline 0 & $81(63.0)$ \\
\hline 1 & $47(37.0)$ \\
\hline \multicolumn{2}{|l|}{ Surgery performed } \\
\hline Complete resection & $64(50.0)$ \\
\hline Partial resection & $64(50.0)$ \\
\hline \multicolumn{2}{|l|}{ Timing of treatment } \\
\hline Neoadjuvant total & $77(60.0)$ \\
\hline GBC & $52(40.0)$ \\
\hline Chemotherapy alone & $40(31.0)$ \\
\hline Chemoradiation & $8(6.0)$ \\
\hline RT alone & $4(3.0)$ \\
\hline CCA & $25(20.0)$ \\
\hline Chemotherapy alone & $20(16.0)$ \\
\hline Chemoradiation & $1(1.0)$ \\
\hline RT alone & $4(3.0)$ \\
\hline No neoadjuvant total & $51(40.0)$ \\
\hline GBC & $38(30.0)$ \\
\hline CCA & $13(10.0)$ \\
\hline
\end{tabular}

Table 1 (continued)
Table 1 (continued)

\begin{tabular}{|c|c|}
\hline Characteristics & Patients, n (\%) \\
\hline \multicolumn{2}{|l|}{ Stage grouping } \\
\hline \multicolumn{2}{|l|}{ Neoadjuvant } \\
\hline GBC & $52(40.0)$ \\
\hline I & $13(10.0)$ \\
\hline II & $21(16.0)$ \\
\hline III & $18(14.0)$ \\
\hline CCA & $25(20.0)$ \\
\hline I & $4(3.0)$ \\
\hline II & $14(11.0)$ \\
\hline III & $7(5.5)$ \\
\hline \multicolumn{2}{|l|}{ No neoadjuvant } \\
\hline GBC & $38(75.0)$ \\
\hline I & $10(7.8)$ \\
\hline II & $10(7.8)$ \\
\hline III & $18(14.0)$ \\
\hline CCA & $13(25.0)$ \\
\hline I & $4(3.0)$ \\
\hline II & $6(4.7)$ \\
\hline III & $3(2.0)$ \\
\hline Radiation treatment (cGy) & $17(13.0)$ \\
\hline Chemoradiation & $9(7.0)$ \\
\hline RT alone & $8(6.0)$ \\
\hline Chemotherapy & $69(54.0)$ \\
\hline Single agent & $44(34.0)$ \\
\hline Multi-agent & $25(20.0)$ \\
\hline
\end{tabular}

GBC, gallbladder cancer; CCA, cholangiocarcinoma. 

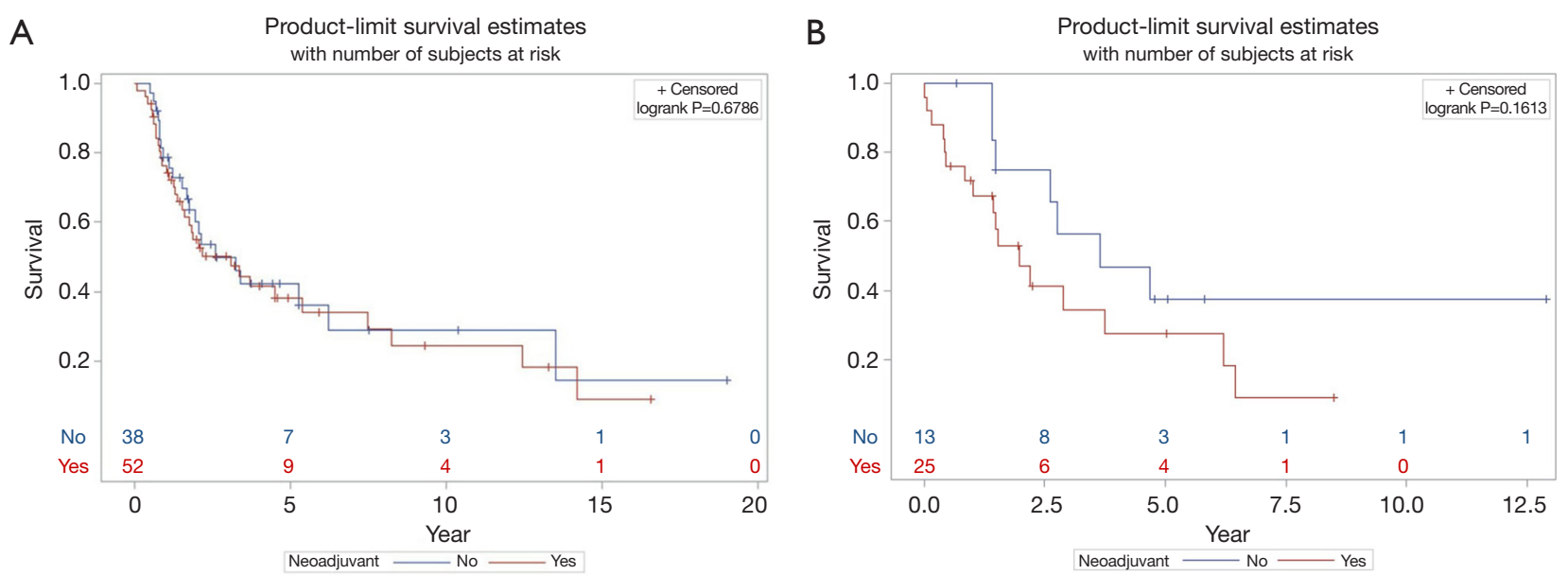

Figure 1 Survival estimates. (A) OS for gall bladder patients; (B) OS for cholangiocarcinoma patients. OS, overall survival.

\section{Results}

We identified 128 patients from our prospectively collected data. Among all patients, 90 (70\%) had GBC and 38 (30\%) had CCA. Median age was 68.5 years and male to female ratio was about 1:2 (45 males and 83 females). Twenty-five patients (27\%) among GBC and 8 patients $(21 \%)$ with CCA were T3, T4 or node positive. ECOG PS was 0 in $81(63 \%)$ and 1 in $47(37 \%)$ of patients. Neoadjuvant treatment was delivered in $77(60 \%)$ patients with $52(40 \%)$ in GBC group and $25(20 \%)$ in CCA patients. Chemotherapy alone was given to 60 patients $(47 \%)$ or radiation with or without chemotherapy to 17 patients (13\%). Forty-four patients $(34 \%)$ received single agent chemotherapy whereas 25 (20\%) patients got multi-agent chemotherapy.

OS was measured as time from date of diagnosis to death from any cause; progression free survival was defined as a time from first day of treatment to disease progression or death from any cause. Response evaluation was done by RECIST criteria (comparing with baseline scan performed not more than one month before the start of treatment). The median OS for GBC patients was 3.1 years with 2.6 years for patients not receiving neoadjuvant treatment versus 3.1 years for neoadjuvant group $(\mathrm{P}=0.6786)$. For CCA patients, median OS was 2.6 years. It was 3.6 years for no neoadjuvant therapy group as compared to 2.0 years for neoadjuvant group $(\mathrm{P}=0.1613)$ (Figure 1). A trend towards increased DF was seen in patients with CCA and GBC receiving neoadjuvant therapy: $\mathrm{HR} 2.74,95 \% \mathrm{CI}$, $0.73-10.36, \mathrm{P}=0.14$ and $0.92,95 \% \mathrm{CI}, 0.44-1.93, \mathrm{P}=0.82$ respectively. The hazard ratio for time to LRF in CCA patients receiving neoadjuvant treatment was $3.17,95 \%$ CI,
0.62-16.31, $\mathrm{P}=0.16$ whereas it was $0.15,95 \% \mathrm{CI}, 0.10-1.76$, $\mathrm{P}=0.23$ for $\mathrm{GBC}$ patients.

The pattern of first failure in patients with GBC was locoregional in $8(10 \%)$ having $3 \mathrm{LRF}$ in neoadjuvant group ( 2 with chemotherapy, 1 with CRT, 0 with RT alone) as compared to 5 in non-neoadjuvant group. In $28(35 \%)$ patients who developed DF as a first site of failure, 15 patients received neoadjuvant therapy whereas 13 patients got non-neoadjuvant treatment. LRF occurred first in 6 patients receiving neoadjuvant treatment ( 3 with chemotherapy, 1 with CRT, 2 with RT alone) as compared to 2 patients who were treated with nonneoadjuvant CRT among CCA patients. DF was the first site of failure in 9 patients treated with neoadjuvant CRT (8 with chemotherapy, 0 with CRT and 1 with RT alone) as compared to 4 patients who did not receive neoadjuvant treatment.

Of the total 128 treated patients, 50 patients were alive at the time of analysis. Fifty-nine patients did not show any evidence of disease at their last follow up.

\section{Discussion}

Advanced stage BTCs, whether metastatic or locally advanced disease, is characterized by local blood vessels involvement and/or extension to bilateral hepatic lobes or with node positivity (10). Some of these patients present with marginally resectable disease. Incomplete (R1/R2) resections in this group lead to outcomes seen in individuals not having surgical resection even with adjuvant chemotherapy and/or radiation $(11,12)$. The 
rationale of neoadjuvant approach for locally advanced or marginally resected BTC is based on the hypothesis that down staging the disease before resection could increase the radical resection ( $\mathrm{R} 0)$ rates and thus the long-term survival. However this approach is not considered standard of care, neither it has been incorporated into any guideline as several reviews have shown its possible negative effect caused by the delay of potential curative surgery $(11,13)$.

In our analysis, there is no difference in survival between patients receiving neoadjuvant and adjuvant therapy in CCA and GBC patients. Both the curves for OS separate after 5 years with no neoadjuvant arms becoming straight lines while neoadjuvant curves dropping down further, implicating that a true difference might be seen after 5 years but indeed with higher sample size in future trials. Furthermore, the curves in CCA patients with or without neoadjuvant treatment never met together at any point which means there might be a difference in these arms but in our study, it didn't reach a statistically significant value. In patients with GBCs, the two lines are intersecting at multiple levels failing to show any significant difference between two arms thus confirming the null hypothesis. Again, it might show improvement in results if sample size is increased. Another important consideration in analysis of this data is that in both graphs, there is vertical line at the end meaning that censoring happened even at the time of analysis.

In advanced BTC patients undergoing medical management only, OS is $2-3$ months, whereas palliative surgical biliary bypass increases it to 6-12 months. It mounts to 12-24 months for resectable cases, which account for $10-35 \%$ of presenting patients (8). As surgical resection with negative margins is considered the only curative option (14), there is sparse data on increased survival with adjuvant therapies. After resection, adjuvant chemotherapy is often employed because of the inherent poor prognostic factors (15). With associated co-morbidity and mortality, the prognosis is dismal even in patients undergoing radical surgery with $>50 \%$ local failure rates in modern series (13).

Analyses of patterns of failure after resection alone are limited with current data suggesting both locoregional and DF in BTCs. LRF is common and underestimated with complications such as biliary obstruction and hepatic failure leading to death. Once patients develop distant disease, they are unlikely to undergo follow up scans or autopsy, thus overlooking the true incidence of LRF. Furthermore, the contribution from persistent locoregional disease to ultimate development of metastatic disease is not known, hence the use of radiation treatment is rational provided the morbidity and mortality associated with patterns of failure in biliary tract malignancies (6).

In this retrospective data set, a trend toward better survival with a $\mathrm{P}$ value of 0.16 was seen in CCA patients who did not receive neoadjuvant treatment, although the same was not observed in GBC patients. This trend was also seen in meta-analysis involving 6,712 BTC patients in whom a benefit of adjuvant therapy was seen in CCA patients with high risk features (16). Recurrence patterns also appear different among the two. This may be due to treatment modality used, patient selection or other unmeasured factors. Concurrent CRT remains pivotal tool in management of BTCs along with definitive surgical treatment, either preoperatively or postoperatively. Careful patient selection for neoadjuvant treatment should be done for better surgical outcomes.

Several studies have considered the efficacy and safety of adjuvant treatment with chemotherapy or chemoradiation in resected BTC. Most of these are retrospective including small number of patients and failed to show any significant benefit $(17,18)$. With few exceptions, these studies are hard to interpret as these combined patients with gallbladder and bile duct cancers which are entirely different entities with different biology. There are conflicting results of adjuvant therapy, with one study showing no significant impact of adjuvant RT in gallbladder patients as opposed to its benefit shown in hilar CCA patients. Distant site initial recurrence was higher in GBCA (85\%) as compared to those with hilar CCA (41\%) (13). On the other hand, Horgan et al. reported a nonsignificant improvement in OS with adjuvant therapy compared with resection alone in meta-analysis of 6,712 patients with no difference between GBCA and CCA patients. Chemotherapy or CRT was associated with statistically greater benefit than RT alone, having greatest benefit in patients with node positive and macroscopic residual disease (R1 resection) (16). After resection, the addition of RT was shown to benefit patients with $\mathrm{T} 2$ or higher stage and node positive disease as shown by a multivariate Cox proportional hazards model for determining individualized survival predictions from adding RT after GBCA surgery $(19,20)$. T1a and T1b, N0 disease can be treated with adjuvant chemotherapy. Phase II data suggest following chemotherapy combinations for BTC. Gemcitabine/oxaliplatin, gemcitabine/capecitabine, capecitabine/cisplatin, capecitabine/oxaliplatin, 5 FU/oxaliplatin, $5 \mathrm{FU} /$ cisplatin and the single agents gemcitabine, capecitabine and $5 \mathrm{FU}$ in the unresectable or 
metastatic setting (21).

The main limitations of this study are its retrospective nature and combining both GBC and CCA patients as one entity to increase the power of study to investigate relevant differences although these are entirely different groups biologically. Despite the challenges associated with accrual of large numbers of patients with BTC for randomized phase III trials, it is widely acknowledged that individual disease sites are evaluated separately. It is interesting that the main endpoint, OS, in this study was not statistically different among the groups but with improved chemotherapy regimens, evolving radiation techniques and accrual of large number of patients in future, trials might show better results.

\section{Conclusions}

In this analysis, we have presented patterns of failure and survival outcomes of a cohort of BTCs treated at an NCI designated cancer center. Although a trend was seen towards better survival in CCA patients who did not receive neoadjuvant chemoradiation, it was not evident in GBC patients. Recurrence patterns were also different among the two, which might be attributed to treatment modality used, patient selection or unmeasured factors.

\section{Acknowledgements}

None.

\section{Footnote}

Conflicts of Interest: The authors have no conflicts of interest to declare.

Ethical Statement: This study was approved by institutional review board of Fox Chase cancer center. As this was a retrospective study, no informed consent has been obtained.

\section{References}

1. Siegel RL, Miller KD, Jemal A. Cancer Statistics, 2017. CA Cancer J Clin 2017;67:7-30.

2. Esnaola NF, Meyer JE, Karachristos A, et al. Evaluation and management of intrahepatic and extrahepatic cholangiocarcinoma. Cancer 2016;122:1349-69.

3. Anderson CD, Pinson CW, Berlin J, et al. Diagnosis and treatment of cholangiocarcinoma. Oncologist 2004;9:43-57.
4. Czito BG, Hurwitz HI, Clough RW, et al. Adjuvant external-beam radiotherapy with concurrent chemotherapy after resection of primary gallbladder carcinoma: a 23-year experience. Int J Radiat Oncol Biol Phys 2005;62:1030-4.

5. de Groen PC, Gores GJ, LaRusso NF, et al. Biliary tract cancers. N Engl J Med 1999;341:1368-78.

6. Glazer ES, Liu P, Abdalla EK, et al. Neither neoadjuvant nor adjuvant therapy increases survival after biliary tract cancer resection with wide negative margins. J Gastrointest Surg 2012;16:1666-71.

7. Nathan H, Pawlik TM, Wolfgang CL, et al. Trends in survival after surgery for cholangiocarcinoma: a 30-year population-based SEER database analysis. J Gastrointest Surg 2007;11:1488-96; discussion 1496-7.

8. Nelson JW, Ghafoori AP, Willett CG, et al. Concurrent chemoradiotherapy in resected extrahepatic cholangiocarcinoma. Int J Radiat Oncol Biol Phys 2009;73:148-53.

9. Lakatos E. Sample sizes based on the log-rank statistic in complex clinical trials. Biometrics 1988;44:229-41.

10. Blechacz B, Gores GJ. Cholangiocarcinoma: advances in pathogenesis, diagnosis, and treatment. Hepatology 2008;48:308-21.

11. Grendar J, Grendarova P, Sinha R, et al. Neoadjuvant therapy for downstaging of locally advanced hilar cholangiocarcinoma: a systematic review. HPB (Oxford) 2014;16:297-303.

12. Witzigmann H, Berr F, Ringel U, et al. Surgical and palliative management and outcome in 184 patients with hilar cholangiocarcinoma: palliative photodynamic therapy plus stenting is comparable to $\mathrm{r} 1 / \mathrm{r} 2$ resection. Ann Surg 2006;244:230-9.

13. Jarnagin WR, Ruo L, Little SA, et al. Patterns of initial disease recurrence after resection of gallbladder carcinoma and hilar cholangiocarcinoma: implications for adjuvant therapeutic strategies. Cancer 2003;98:1689-700.

14. Wolpin BM, Mayer RJ. A step forward in the treatment of advanced biliary tract cancer. N Engl J Med 2010;362:1335-7.

15. Murakami $Y$, Uemura K, Hayasidani $Y$, et al. Indication for postoperative adjuvant therapy in biliary carcinoma based on analysis of recurrence and survival after surgical resection. Dig Dis Sci 2009;54:1360-4.

16. Horgan AM, Amir E, Walter T, et al. Adjuvant therapy in the treatment of biliary tract cancer: a systematic review and meta-analysis. J Clin Oncol 2012;30:1934-40.

17. Aloia TA, Jarufe N, Javle M, et al. Gallbladder cancer: 
expert consensus statement. HPB (Oxford) 2015;17:681-90.

18. Chan E, Berlin J. Biliary tract cancers: understudied and poorly understood. J Clin Oncol 2015;33:1845-8.

19. Wang SJ, Fuller CD, Kim JS, et al. Prediction model for estimating the survival benefit of adjuvant radiotherapy for gallbladder cancer. J Clin Oncol 2008;26:2112-7.

Cite this article as: Fareed MM, DeMora L, Esnaola NF, Denlinger CS, Karachristos A, Ross EE, Hoffman J, Meyer JE. Concurrent chemoradiation for resected gall bladder cancers and cholangiocarcinomas. J Gastrointest Oncol 2018;9(4):762768. doi: 10.21037/jgo.2018.05.09
20. Wang SJ, Lemieux A, Kalpathy-Cramer J, et al. Nomogram for predicting the benefit of adjuvant chemoradiotherapy for resected gallbladder cancer. J Clin Oncol 2011;29:4627-32.

21. Hezel AF, Zhu AX. Systemic therapy for biliary tract cancers. Oncologist 2008;13:415-23. 